\title{
Defining Cost Functions and Profitability Measures for Digraphs Associated with Raster DEMs
}

\author{
Pascal Matsakis ${ }^{* \dagger}$, Julien Gadiou*, Jacky Desachy* \\ matsakis@irit.fr \\ "Université Paul Sabatier - IRIT \\ 118 , route de Narbonne \\ 31062 Toulouse Cedex, France \\ ${ }^{\dagger}$ CRIL Ingenierie - Groupe Coritec \\ 5 , avenue Marcel Dassault \\ 31500 Toulouse, France
}

\begin{abstract}
With a raster Digital Elevation Model, it is usual to associate a directed graph. Firstly, the problem of defining cost functions for such digraphs is discussed in a general and formal framework, and a particularly simple and natural way to tackle this problem is proposed. Secondly, the notion of profitability, which is commonly linked with the notion of cost, is put forward. Thus, profitability measures are introduced. In particular, the profitability of a point according to a region is defined. Finally, it is shown that profitability measures and cost functions provide complementary information.
\end{abstract}

Keywords. DEMs, directed graphs, cost functions, problems of optimal paths.

\section{Introduction}

With a raster DEM, it is usual to associate a digraph. To each path of this digraph a cost may be attached. Typically, a DEM represents a part of the surface of the earth. A path of the associated digraph then corresponds to a path on the surface. And the cost of the digraph path may correspond to the (euclidean) length of the surface path. It may also correspond to a time or a gasoline consumption. In this paper, only digraphs associated with raster DEMs are considered. Firstly, the problem of defining cost functions for these graphs is discussed in a general and formal framework. A particularly simple and natural way to tackle this problem is proposed in section $\S 2$. The presented approach develops from euclidean to discrete geometry. Thus, cost functions defined on paths of the affine euclidean space are introduced. As shown in $\S 4$, cost functions of graphs are to space cost functions, as a raster DEM is to the represented surface. Secondly, the notion of profitability is put forward. It is in the habit of saying that such a place is near and of easy access, or near but inaccessible, distant but quite accessible, etc. Distance and accessibility can thereby be complementary criteria for the research of particular spots, for instance within the framework of regional development. Profitability is to cost as accessibility is to distance. Profitability measures on paths of the affine euclidean space are introduced in $\S 3$. As shown in $\$ 4$, they enable to define on a DEM the profitability of a point according to a region. By way of conclusion, $\$ 5$, experimental results illustrate how profitability measures and cost functions provide complementary information. Note that $\mathbf{N}$ is the set of natural numbers, $\mathbf{Z}$ is the one of relative numbers and $\mathbf{R}$ the real numbers one. $a . b$, where $a$ and $b$ are relative numbers, is $\{n \in Z / a \leq n \leq b\}$ and $\overline{\mathbf{R}}$ is $\mathbf{R} \cup\{-\infty,+\infty\}$. 


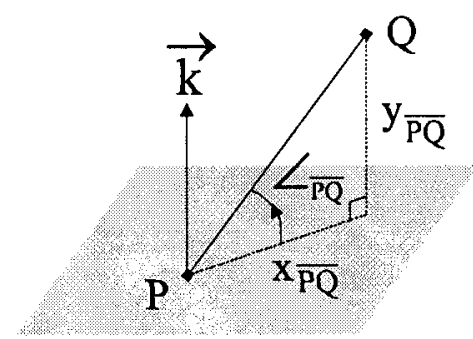

Fig. 1. Linear paths of $\mathcal{C}$.

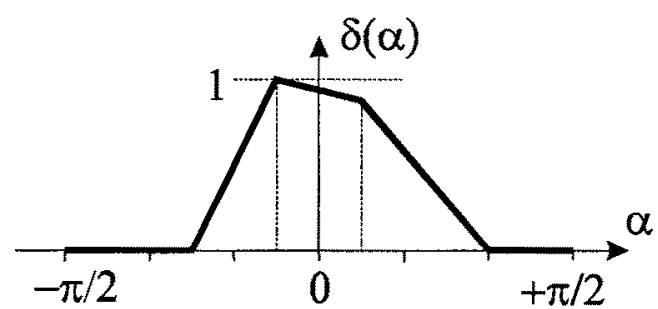

Fig. 2. Generating a hill-climbing energetic function. The hill-climber feels mostly at ease on a $15^{\circ}$ slope. He refuses slopes up over $60^{\circ}$ and slopes down over $45^{\circ}$.

\section{Space Cost Functions}

\subsection{Terminology and Notations}

The space is a directed affine euclidean space referred to the direct orthonormal frame $(0, \vec{i}, \vec{j}, \vec{k})$. The plane is the affine subspace referred to the direct frame $(\mathrm{O}, \vec{i}, \vec{j})$. In this section $\S 2$, a path is an oriented geometrical arc of space. The length of a path is its euclidean length. A linear path is a path of space without double point and whose support is a segment. $\overline{P_{1} P_{2}}$, where $P_{1}$ and $P_{2}$ are two points of space, denotes the linear path joining $P_{1}$ to $P_{2}$. Let $n$ be an integer such that $n \geq 3$, $\left(P_{i}\right)_{i \in 1 . n n}$ a sequence of $n$ points of space. $\overline{P_{i}} \bar{i} \in 1 . . n$ denotes the path obtained by juxtaposition of the arcs $\overline{P_{i}} i \in 1 . n-1$ and $\overline{P_{n-1} P_{n}}$. Its length is $\Sigma_{i \in 1 . . n-1} P_{i} P_{i+1}$. Now consider the set of the paths which benefit by the recurrent notation that has been introduced. $C$ will represent the part of this set including following paths only: $\overline{P_{i}}{ }^{i \in L_{n} n}$ such that for any element $i$ of $1 . . n-1$ the orthogonal projections of $P_{i}$ and $P_{i+1}$ onto the plane are distinct. From now on, the term path will be applied to the elements of $C$ only. Let $\overline{\mathrm{PQ}}$ be a path of $\mathrm{C}$ (more precisely, "let $\mathrm{P}$ and $\mathrm{Q}$ be two points of space such that: $\left.\overrightarrow{\mathrm{PQ}} \in \mathrm{C}^{\prime \prime}\right)$. Let $\vec{u}$ be the orthogonal projection of $\overrightarrow{\mathrm{PQ}}$ onto the plane $(\vec{i}, \vec{j})$ and let $\vec{v}$ be the unit vector $\vec{u}|| \vec{u} \mid$. Defined relatively to the direct vector plane $(\vec{v}, \overrightarrow{\mathrm{k}}): \angle \overrightarrow{\mathrm{PQ}}$ is the measure of the oriented angle between $\vec{v}$ and $\overrightarrow{P Q}, x \overline{P Q}$ and $y \overline{P Q}$ are the coordinates of $\overrightarrow{\mathrm{PQ}}$ (Fig. 1$). \angle \overline{\mathrm{PQ}} \in]-\pi / 2, \pi / 2\left[\right.$ and $\mathrm{x} \overline{\mathrm{PQ}} \in \mathbf{R}_{+}^{*}$ and $\mathrm{y} \overline{\mathrm{PQ}} / \mathrm{x} \overline{\mathrm{PQ}}=\tan (\angle \overline{\mathrm{PQ}})$. $\angle \overline{\mathrm{PQ}}$ measures the angle between the $\overline{\mathrm{PQ}}$ and the plane. It is the slope of the path.

\subsection{Slope-Dependent Cost Functions}

Definition 1. A space cost function is a map $C$ from $C$ into $\overrightarrow{\mathbf{R}}_{+}^{*}$ satisfying [A1]: [A1] Let $\overline{P_{i}} i \in 1 . . n$ be a path of $C: C\left(\bar{P}_{i} i 1_{1 . n n}\right)=\Sigma_{i \in 1, . n-1} C\left(\overline{P_{i} P_{i+1}}\right)$. Given $\mu$ a path of $C$. $C(\mu)$ is the cost of $\mu$. If $C(\mu)$ is finite, $\mu$ is potentially profitable.

Any path of $\mathrm{C}$ hence admits a cost ( $\mathrm{C}$ is a map) and may absolutely not be profitable (a cost may be infinite). Moreover, travelling is always costly (a cost is a strictly positive value). Note that certain problems of optimal paths call for replacing the sum calculation in [A1] by the calculation of the minimum [Gon84] or of the average [Ahu93], or even of the product [Pri94]. And the matter may be then to research maxcost paths and no more min-cost paths. The previous definition is however adapted to the majority of the practically encountered problems. 
Definition 2. A space cost function $\mathrm{C}$ is said to be slope-dependent iff it satisfies [A2]: [A2] Let $\overline{\mathrm{PQ}}$ and $\overline{\mathrm{RS}}$ be two paths of $\mathrm{C}:[\mathrm{PQ}=\mathrm{RS}$ and $\angle \overline{\mathrm{PQ}}=\angle \overline{\mathrm{RS}}] \Rightarrow \mathrm{C}(\overline{\mathrm{PQ}})=\mathrm{C}(\overline{\mathrm{RS}})$.

The slope and length of a linear path then determine its cost. In other words, the exact localization of the path in space is not important: space is considered homogeneous. In practice, it is far from being always the case. For instance, a $4 \times 4$ vehicle can travel on road or on uneven ground, on dry or sodden soil, through a thick or scattered vegetation, along or against the wind direction, etc. ([Mi191], [Zha93], [Kre94], [Dub95]...). Slope-dependent cost functions are then not adapted to cost modelization (supposing that the available data do not only consist in topographical ones!). However that may be, these are fundamental functions because the cost function associated with a non-homogeneous space can be defined from a parametrized family of slopedependent functions (one corresponding for instance to the travel on tarred road, the other on stony path, etc.). Moreover, the profitability measures (see $\$ 3$ ) need to be based on such cost functions, representing ideal spaces. Proposition 1 expresses that the cost of a linear path of given slope is proportional to the length of this path.

Proposition 1. Let $\mathrm{C}$ be a slope-dependent cost function, $\overline{\mathrm{PQ}}$ and $\overline{\mathrm{RS}}$ two paths of $\mathrm{C}$ and $\mathrm{k}$ a strictly positive real number: $[\mathrm{PQ}=\mathrm{k} \cdot \mathrm{RS}$ and $\angle \overline{\mathrm{PQ}}=\angle \overline{\mathrm{RS}}] \Rightarrow \mathrm{C}(\overline{\mathrm{PQ}})=\mathrm{k} \cdot \mathrm{C}(\overline{\mathrm{RS}})$

\subsection{Generator of a Space Cost Function}

Definition 3. Let $\theta$ be an element of ]- $\pi / 2, \pi / 2$ [ and let $C$ be a slope-dependent space cost function. $C$ is said to be minimal at $\theta$ iff it satisfies the following properties:

[A3] Let $\overline{\mathrm{PQ}}$ and $\overline{\mathrm{RS}}$ be two paths of $\mathrm{C}:(\mathrm{PQ}=\mathrm{RS}$ and $\angle \overline{\mathrm{RS}}=\theta) \Rightarrow \mathrm{C}(\overline{\mathrm{PQ}}) \geq \mathrm{C}(\overline{\mathrm{RS}})$ [A4] Let $\overline{\mathrm{RS}}$ be a path of $\mathrm{C}:[\mathrm{RS}=1$ and $\angle \overline{\mathrm{RS}}=\theta] \Rightarrow \mathrm{C}(\overline{\mathrm{RS}})=1$

From [A3], it derives that among all linear paths with length 1, those with slope $\theta$ have the lowest cost. [A4] sets this minimal cost to 1 . The only real contribution of [A4] is to guarantee the existence of potentially profitable paths. Remark that the map from $C$ into $\overline{\mathbf{R}}_{+}^{*}$ which associates each path with its length is minimal at any element of $]-\pi / 2, \pi / 2[$.

Proposition 2. $\Rightarrow$ Let $\theta$ be an element of $]-\pi / 2, \pi / 2[$ and let $C$ be a slope-dependent space cost function. If $C$ is minimal at $\theta$, there exists a map $\delta$ from $]-\pi / 2, \pi / 2$ [ into $[0,1]$ such that for any $\overline{\mathrm{PQ}}$ of $\mathrm{C}: \mathrm{C}(\overline{\mathrm{PQ}})=\mathrm{x} \overline{\mathrm{PQ}} /[\cos (\angle \overline{\mathrm{PQ}}) \cdot \delta(\angle \overline{\mathrm{PQ}})]$. This map $\delta$ is unique and takes the value 1 at $\theta$. $\Leftarrow$ Let $\theta$ be an element of $]-\pi / 2, \pi / 2[$ and let $\delta$ be a map from $]-\pi / 2, \pi / 2[$ into $[0,1]$ taking the value 1 at $\theta$. There exists a space cost function $\mathrm{C}$ such that for any path $\overline{\mathrm{PQ}}$ of $\mathrm{C}: \mathrm{C}(\overline{\mathrm{PQ}})=\mathrm{x} \overline{\mathrm{PQ}} /[\cos (\angle \overrightarrow{\mathrm{PQ}}) \cdot \delta(\angle \overline{\mathrm{PQ}})]$. This cost function is unique, slope-dependent and minimal at $\theta$.

Definition 4. Let $\theta$ be an element of ]- $\pi / 2, \pi / 2$ [. According to proposition 2, the datum of a space cost function, slope-dependent and minimal at $\theta$, is equivalent to the one of a map $\delta$ from $]-\pi / 2, \pi / 2[$ into $[0,1]$ taking the value 1 at $\theta: \delta$ is the cost function generator.

$\delta(\alpha)$, for any element $\alpha$ of $]-\pi / 2, \pi / 2[$, is the distance a cost unit enables to cover on a linear path of slope $\alpha$. The interest of expressing $C$ in terms of $\delta$ lies in this simple interpretation. From a practical point of view, defining a cost function by means of its generator is particularly convenient and natural (Fig.2). 


\subsection{A Typical Family of Space Cost Functions}

Definition 5. Let $\theta$ be an element of ]- $\pi / 2, \pi / 2[$ and $\mathrm{C}$ a space cost function, slope-dependent and minimal at $\theta$. $C$ is called a hill-climbing energetic function iff it satisfies [A5]: [A5] Let $\overline{\mathrm{PQ}}$ and $\overline{\mathrm{RS}}$ be two paths of $\mathrm{C}$ :

$$
[\mathrm{PQ}=\mathrm{RS} \text { and }(\angle \overline{\mathrm{PQ}} \leq \angle \overline{\mathrm{RS}} \leq \theta \text { or } \theta \leq \angle \overline{\mathrm{RS}} \leq \angle \overline{\mathrm{PQ}})] \Rightarrow \mathrm{C}(\overline{\mathrm{PQ}}) \geq \mathrm{C}(\overline{\mathrm{RS}})
$$

A hill-climber gets less tired on a linear path of slope $\theta$. The further from $\theta$ the slope is (i.e. the more abrupt the slope up or the steeper the slope down), the more energy is consumed. The idea is to penalize abrupt ways (Fig.2). The map from $C$ into $\overline{\mathbf{R}}_{+}^{*}$ which associates each path with its length is a hill-climbing energetic function.

\section{Space Profitability Measures}

In this section, profitability measures on paths of the space are introduced. The calculation of the profitability of a path joining $P$ to $Q$ is based on the estimate, drawn from a priori knowledge, of the travel cost from $\mathrm{P}$ to $\mathrm{Q}$. The knowledge at stake are voluntarily limited: for instance to the position of the orthogonal projections of $\mathrm{P}$ and $\mathrm{Q}$ onto the plane, or to the position of $\mathrm{P}$ and $\mathrm{Q}$ in space, the length of the min-cost path from $\mathrm{P}$ to $\mathrm{Q}$, etc. Each case leads to a particular profitability measure. As illustrations, two measures are briefly described here. $C$ denotes a space cost function, $D_{\mathrm{P}}$ and $\mathrm{D}_{\mathrm{Q}}$ the lines directed by $\overrightarrow{\mathrm{k}}$ and running through $\mathrm{P}$ and $\mathrm{Q}, \mathrm{P} @ \mathrm{Q}$ the set of paths belonging to $C$ and joining $P$ to $Q, D_{P} @ D_{Q}$ the set of paths belonging to $C$ and joining one point of $D_{P}$ to one point of $D_{Q}: D_{P} @ D_{Q}=\underset{\left(P^{\prime}, Q^{\prime}\right) \in D_{p} \times D_{Q}}{U} P^{\prime} @ Q^{\prime}$

\subsection{The 2D-Profitability Measure}

Definition 6. The 2D-profitability measure is a function $\mathrm{A}^{2 \mathrm{D}}$ from $C$ into $[0,1]$. Let $\mu$ be a path of $C$ joining a point $P$ to a point $Q . A^{2 D}$ is defined at $\mu$ iff inf $v \in D_{p} @ D_{Q} C(v)$ is finite (it is especially the case when $\mu$ is potentially profitable). It is then set that:

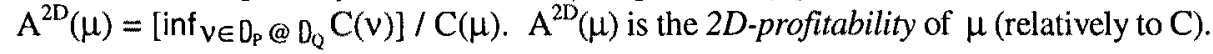

Forecasting to spend inf $v \in D_{P} @ D_{Q} C(v)$ to join $Q$ from $P$, means at the same time to be economical, pragmatic, very optimistic and (really) misinformed. As if a hill-climber, in order to assess the distance he still has to cover, would draw a segment on a rudimentary touristic map and consider the relief to be certainly as he hopes to be. So is $\mathrm{A}^{2 \mathrm{D}}$. And its judgement gets more severe: if somebody is advised to follow $\mu$ to get from $P$ to $Q$, the measure will probably assess that, comparing $C(\mu)$ with the cost initially forecast, the suggestion was not the best (and even dishonest). In the case where $\mathrm{C}$ is the map from C into $\overline{\mathbf{R}}_{+}^{*}$ which associates each path with its length, the forecast cost is the distance between lines $D_{P}$ and $D_{Q}$, i.e. $x_{\overline{P Q}}$. Consequently, measure $A^{2 D}$ is a map and value $A^{2 D}(\mu)$ is simply $\times \overline{P Q} / \lambda$ - where $\lambda$ denotes the length of $\mu$. The following proposition gives a practical means to characterize $\mathrm{A}^{2 \mathrm{D}}$ in a more general case.

Proposition 3. If the cost function $C$ is generated by a continuous map $\delta$, the profitability measure $A^{2 D}$ is an everywhere defined measure. Moreover, for any couple $(P, Q)$ of points: $\left[\overline{\mathrm{PQ}} \in \mathrm{C} \Rightarrow \inf _{\mathrm{v} \in \mathrm{D}_{\mathrm{P}} @ \mathrm{D}_{\mathrm{Q}}} \mathrm{C}(\mathrm{v})=\mathrm{X}_{\overline{\mathrm{PQ}}}{ }^{\prime} \max _{\alpha \in\} \pi / 2, \pi / 2[}(\delta(\alpha) \cdot \cos (\alpha))\right]$ and $\left[\overline{\mathrm{PQ}} \notin C \Rightarrow \inf _{\mathrm{v} \in \mathrm{O}_{\mathrm{p}} @ 0_{\mathrm{Q}}} \mathrm{C}(\mathrm{v})=0\right]$ 


\subsection{The 3D-Profitability Measure}

Definition 7. The $3 D$-profitability measure is a function $\mathrm{A}^{3 \mathrm{D}}$ from $\mathrm{C}$ into $[0,1]$. Let $\mu$ be a path of $C$ joining a point $P$ to a point $Q$. $A^{3 D}$ is defined at $\mu$ iff inf $v \in P @ Q C(v)$ is finite (it is especially the case when $\mu$ is potentially profitable). It is then set that: $\mathrm{A}^{3 \mathrm{D}}(\mu)=\left[\inf _{v \in \mathrm{P} @ \mathrm{Q}} \mathrm{C}(v)\right] / \mathrm{C}(\mu) \mathrm{A}^{3 \mathrm{D}}(\mu)$ is the $3 D$-profitability of $\mu$ (relatively to $\mathrm{C}$ ).

Forecasting to spend inf $f_{v \in P @ Q} C(v)$ to join $Q$ from $P$, means at the same time to be economical, pragmatic, very optimistic and (rather) misinformed. As if a motorist, in order to assess the distance he still has to cover, would scan an ordnance map and consider that tunnels have certainly been excavated and bridges erected. $\mathrm{A}^{3 \mathrm{D}}$ sticks more to realities than $\mathrm{A}^{2 \mathrm{D}}$. Its judgement gets less severe. If $\mathrm{C}$ associates each path with its length, then the forecast cost is $P Q$. In the general case, inf $v \in P @ Q C(v)$ may be difficult to calculate. This point will not be tackled here.

\section{Back to the Discrete Space}

After a quick reminder about cost functions of graphs and optimal paths, in $\S 4.1$, it is shown in $\$ 4.2$ how to associate a weighted digraph with a raster DEM and a space cost function. Moreover, in $\S 4.3$, profitability measures are defined on the graph vertices.

\subsection{Cost Functions of Graphs and Optimal Paths}

Let $(X, U, V)$ be any weighted and directed graph. Assume that $V$ is a map from $U$ into $\overline{\mathbf{R}}_{+}^{*}$. The function from the set of graph paths into $\overline{\mathbf{R}}_{+}^{*}$, which associates each path $\left(\mathrm{u}_{\mathrm{i}}\right)_{\mathrm{i} \in \mathrm{1} . . \mathrm{n}}$ with the value $\Sigma_{\mathrm{i} \in 1 . . \mathrm{n}} \mathrm{V}\left(\mathrm{u}_{\mathrm{i}}\right)$, is the cost function of $(\mathrm{X}, \mathrm{U}, \mathrm{V}) . \Sigma_{\mathrm{i} \in \mathrm{L} . . \mathrm{n}} \mathrm{V}\left(\mathrm{u}_{\mathrm{i}}\right)$

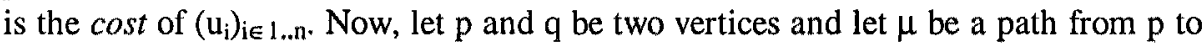
q. If the cost of any path from $\mathrm{p}$ to $\mathrm{q}$ is greater than or equal to the cost of $\mu$ then $\mu$ is an optimal path - or a min-cost path - from p to q. The cost of $\mu$ is the min-cost to reach $q$ from $\mathrm{p}$. Finally, let $\Delta$ be the function from $\mathrm{X}^{2}$ into $\overline{\mathbf{R}}_{+}^{*}$ which associates each couple (p,q) with the min-cost to reach $q$ from $p$, let $Y$ be a non-empty subset of $X$ and let $\Delta_{\mathrm{Y}}$ be the function from $\mathrm{X}$ into $\overline{\mathbf{R}}_{+}^{*}$ defined by: $\forall \mathrm{p} \in \mathrm{X}, \Delta_{\mathrm{Y}}(\mathrm{p})=\min \mathrm{q} \in \mathrm{Y} \Delta(\mathrm{q}, \mathrm{p})$. $\Delta(q, p)$ is the min-cost to reach $p$ from $q$. A path from $q$ to $p$ is optimal iff its cost is $\Delta(q, p)$. $\Delta_{\mathrm{Y}}(\mathrm{p})$ is the min-cost to reach $\mathrm{p}$ from $\mathrm{Y} . \Delta_{\mathrm{Y}}$ is the min-cost function according to $\mathrm{Y}$.

\subsection{Weighted Digraphs, Raster DEMs and Space Cost Functions}

With a numerical image, it is usual to associate the directed graph whose vertices are the image pixels and the arcs are, generally, the couples of 8-adjacent vertices. Let hence I be a raster DEM and let $(\mathrm{X}, \mathrm{U})$ be the digraph associated with I. The pixels of $I$ are assimilated to points of the discrete space $\mathbf{Z}^{2}$. This space itself is embedded into the affine euclidean $x y-p l a n e$. The choice of $U$ incites to provide $\mathbf{Z}^{2}$ with a discrete distance, and more precisely with a ponderate distance $d$ defined by a $3 \times 3$ mask. In practice, in order to approach the euclidean distance, the chamfer distance 3-4 is generally recommended [Bor86]. Now, it is considered that I is a raster model of a surface of the affine euclidean space. To any pixel of I consequently corresponds a point of this surface. Pixel and associated point will be named by the same letter: 
small for the first and capital for the second. To pixel p of I thus corresponds a point $P$ of the affine space: $p$ is the orthogonal projection of $P$ onto the $x y$-plane and the $z-$ coordinate of $\mathrm{P}$ is the gray-level of $\mathrm{p}$ in I (up to a scale factor). Finally, let $\mathrm{C}$ be a space cost function, as defined in $\$ 2$. C enables to weight the digraph $(X, U)$ by means of the map $V$ from $U$ into $\overline{\mathbf{R}}_{+}^{*}$ which associates each $\operatorname{arc}(p, q)$ with $C(\overline{P Q})$ (we will hark back to this point in $\S 4.3$ ). Consider a path $\mu$ of the weighted digraph

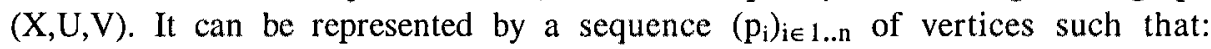
$\forall \mathrm{i} \in 1 . . \mathrm{n}-1,\left(\mathrm{p}_{\mathrm{i}}, \mathrm{p}_{\mathrm{i}+1}\right) \in \mathrm{U}$. The cost of $\mu$ is the value $\Sigma_{\mathrm{i} \in 1 . . \mathrm{n}-1} \mathrm{~V}\left(\mathrm{p}_{\mathrm{i}}, \mathrm{p}_{\mathrm{i}+1}\right)$, or also

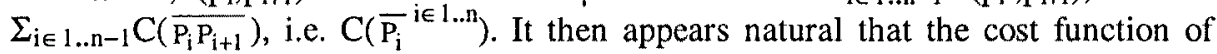
the graph, as well as the space cost function, should be denoted $C$.

\subsection{Profitability of a Point According to a Region}

Let $I$ be a raster DEM, $C$ a space cost function and $(X, U, V)$ the graph associated with $(I, C)$. Consider a non-empty subset $Y$ of $X$. Here will be defined a function from $\mathrm{X}$ into $[0,1]$ called profitability measure according to $\mathrm{Y}$. The notion of space profitability measure, developed in $\$ 3$, will of course contribute to this end. Within the framework of this paper, it will exclusively be referred to measure $A^{2 D}$. Moreover, it will from now on be supposed that $C$ is generated by a continuous map $\delta$. Consider, for a given vertex $p$ of $X-Y$, the expression: $\min _{q \in Y}\left[\right.$ inf $\left._{v \in D_{Q} @ D_{p}} C(v)\right]$. As a direct extension of $\$ 3.1$, it appears natural to interpret this value as the cost forecasted by $\mathrm{A}_{\mathrm{Y}}^{2 \mathrm{D}}$ to reach $\mathrm{p}$ from $\mathrm{Y}$ - where $\mathrm{A}_{\mathrm{Y}}^{2 \mathrm{D}}$ denotes the profitability measure we want to define. It also appears natural to welcome $p$ in the definition domain of $A_{Y}^{2 D}$ iff this cost is finite. In this case: $A_{Y}^{2 D}(p)=\left(\min _{q \in Y}\left[\inf _{v \in D_{Q} @ D_{p}} C(v)\right]\right) / \Delta_{Y}(p)$. The path that will be "really taken" is indeed the optimal path, whose cost is $\Delta_{Y}(p)$. Now, according to proposition 3: inf $\left.v \in D_{Q} @ D_{p} C(v)=q p / \max \alpha \in\right]-\pi / 2, \pi / 2[(\delta(\alpha) \cdot \cos (\alpha))$. Where $q p$ is obviously the euclidean distance between $q$ and $p$. Consequently:

$$
\min _{\mathrm{q} \in Y}\left[\inf _{v \in D_{Q} @ D_{P}} C(v)\right]=\left(\min _{q \in Y} q p\right) / \max _{\alpha \in]-\pi / 2, \pi / 2[}(\delta(\alpha) \cdot \cos (\alpha))
$$

The discrete transcription of the numerator is $\min \underset{q \in Y}{ } d(q, p)$ or also $d_{Y}(p)$ - by denoting $d_{Y}$ the distance image according to $\mathrm{Y}$ (remember that $\mathrm{d}$ is a chamfer distance, see $\$ 4.2$ ). For obvious practical reasons, it is tempting to adopt it. But, to this end, the discrete transcription of the cost calculation must be operated. In $\$ 4.2$, we had weighted each $\operatorname{arc}(\mathrm{p}, \mathrm{q})$ by $\mathrm{C}(\overline{\mathrm{PQ}})$, i.e., according to proposition 2 , by: $\mathrm{C}(\overline{\mathrm{PQ}})=\mathrm{x} \overline{\mathrm{PQ}} /[\cos (\angle \overline{\mathrm{PQ}}) \cdot \delta(\angle \overline{\mathrm{PQ}})]$. Coming back to the definition of $\mathrm{V}$, we set:

$$
\forall(\mathrm{p}, \mathrm{q}) \in \mathrm{U}, \mathrm{V}(\mathrm{p}, \mathrm{q})=\mathrm{d}(\mathrm{p}, \mathrm{q}) /[\cos (\angle \overline{\mathrm{PQ}}) \cdot \delta(\angle \overline{\mathrm{PQ}})]
$$

Remark that $\mathrm{C}(\mathrm{p}, \mathrm{q})$ - where $\mathrm{C}$ denotes the cost function of the digraph - is not exactly equal to $\mathrm{C}(\overline{\mathrm{PQ}})$ any more - where $\mathrm{C}$ now denotes the space cost function.

Definition 8. The 2D-profitability measure $\mathrm{A}_{\mathrm{Y}}^{2 \mathrm{D}}$ according to $\mathrm{Y}-$ or $2 D$-profitability image according to $\mathrm{Y}$ - is the map from $\mathrm{X}$ into $[0,1]$ which takes the value

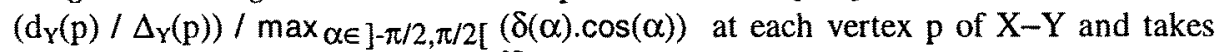
the value 1 at each vertex of $\mathrm{Y} . \mathrm{A}_{\mathrm{Y}}^{2 \mathrm{D}}(\mathrm{p})$ is the $2 D$-profitability of $\mathrm{p}$ according to $\mathrm{Y}$.

If $\mathrm{C}$ is the hill-climbing energetic function which associates each path of $\mathrm{C}$ with its euclidean length, then $\mathrm{A}_{\mathrm{Y}}^{2 \mathrm{D}}$ is defined on $\mathrm{X}$ by: $\forall \mathrm{p} \in \mathrm{X}, \mathrm{A}_{\mathrm{Y}}^{2 \mathrm{D}}(\mathrm{p})=\mathrm{d}_{\mathrm{Y}}(\mathrm{p}) / \Delta_{\mathrm{Y}}(\mathrm{p})$. 
Gaussian Hill. Costs and profitabilities are according to the upper-left corner.

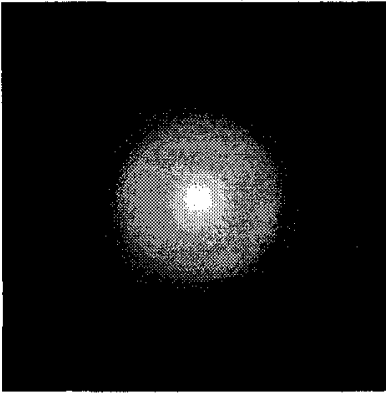

DEM

highest point: 120 gray levels

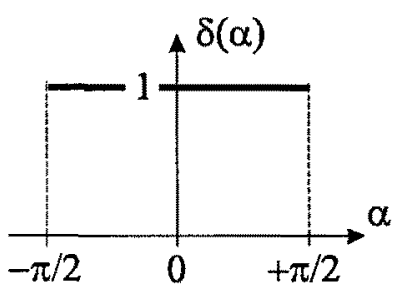

$\alpha$

$-\pi / 2 \quad 0+\pi / 2$

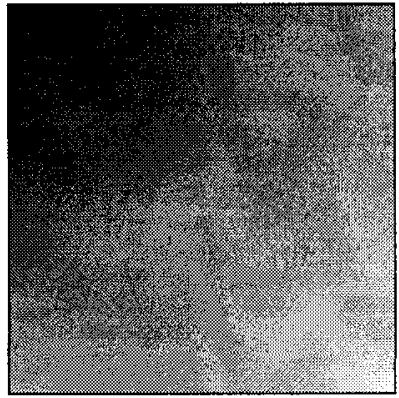

min-cost image

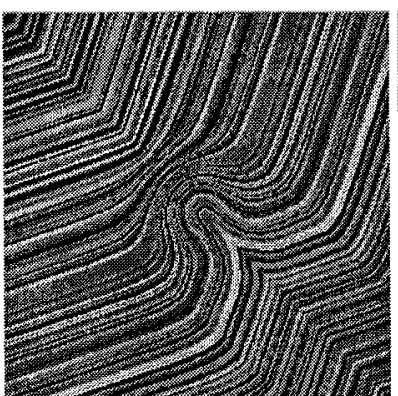

random LUT

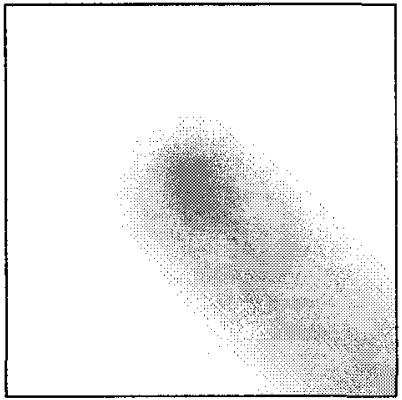

profitability image minimal profitability: 0.67

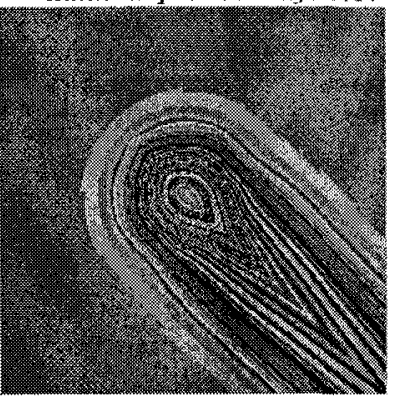

random LUT

Mont Ventoux (France). Covered surface: $100 \mathrm{~km}^{2}$. Maximal difference in level: $600 \mathrm{~m}$.

Costs and profitabilities are according to a point situated amid the upper part of the image.

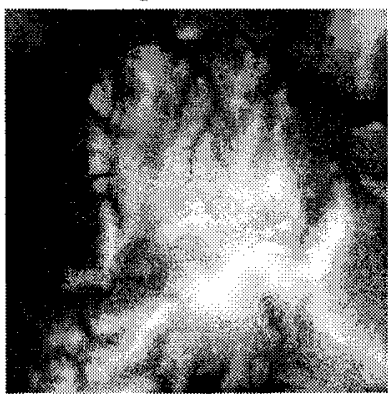

DEM

equalized histogram

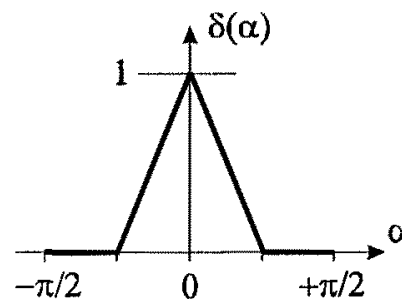

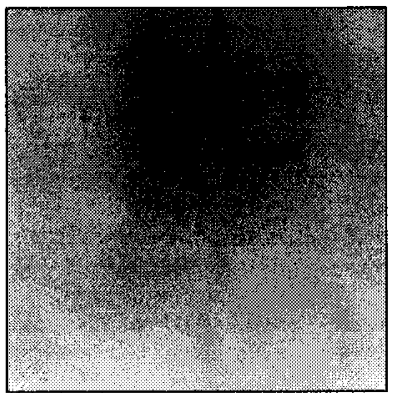

min-cost image

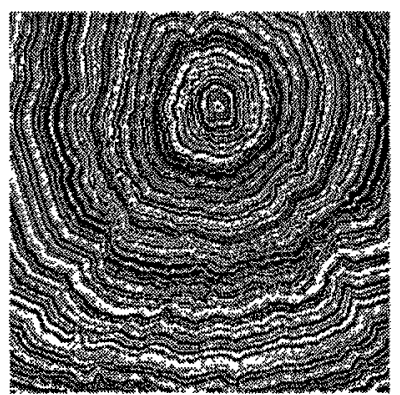

random LUT

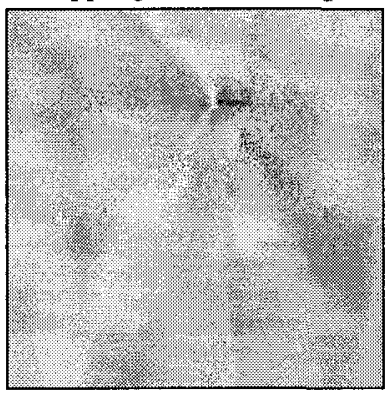

profitability image minimal profitability: 0.45

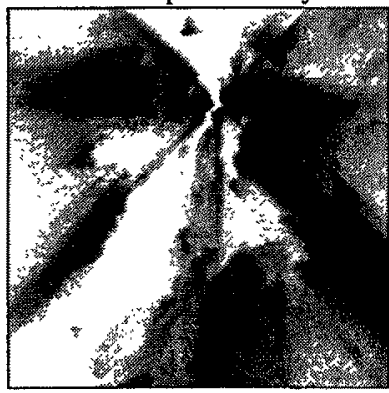

equalized histogram 


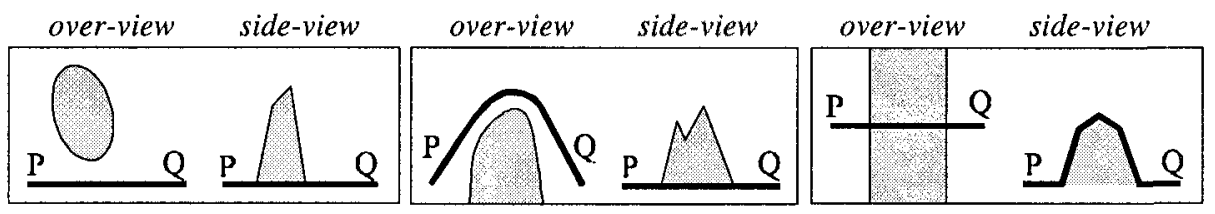

Fig. 3. 2D-profitability measures. Some characteristics.

The represented paths are the optimal paths from $P$ to $Q$. In the first case (on the left), point $Q$ may be assessed totally profitable according to $\{\mathrm{P}\}$. Depending on $\delta, Q$ may be assessed more profitable in the second case than in the third one, even if the $P Q$ distances are identical and also the lengths of the optimal paths.

\section{Experimental Results and Conclusion}

In this paper, the problem of defining cost functions for digraphs associated with raster DEMs has been discussed in a general and formal framework. A particularly simple and natural way to tackle this problem has been proposed. Moreover, the notion of profitability has been put forward and profitability measures have been defined. The calculation of profitabilities is based on the datum of a space cost function - representing an ideal homogeneous space - and consists in drawing estimates from a priori knowledge of travel costs. Profitability measures and cost functions provide useful and complementary information. The results of two experiments are given here in order to illustrate this point. The min-cost images $\Delta_{Y}$ have been computed by means of the well-known Bellman's algorithm [Bel58] and the distance images $\mathrm{d}_{\mathrm{Y}}$ by means of a very efficient algorithm [Ros66] [Bor84] which needs exactly two passes over the data set. High elevations, profitabilities and costs are represented in light gray. All images are $256 \times 256$.

\section{References}

[Ahu93] Ahuja, R.K., T.L. Magnanti, and J.B. Orlin, Network flows, Prentice Hall, 1993.

[Bel58] Bellman, R., "On a toutin problem", Quaterly of Applied Math., 16(1), pp.87-90, 1958.

[Bor84] Borgefors, G., "Distance transformations in arbitrary dimensions", CVGIP 27, pp.321-345, 1984.

[Bor86] Borgefors, G.,"Distance transformations in digital images", CVGIP 34, pp.344-371, 1986.

[Dub95] Dubois, N., F. Semet, "Estimation and determination of shortest path length in a road network with obstacles", European Journal of Operational Research 83,pp. 105-116, 1995.

[Gon84] Gondran, M., and M. Minoux, Graphs and Algorithms, Wiley, Chichester, 1984.

[Kre94] van Kreveld, M., "On Quality Paths on Polyhedral Terrains", IGIS'94, LNCS 884, Springer-Verlag, pp.113-122, 1994.

[Mit91] Mitchell, J.S.B., C.H. Papadimitriou, "The weighted region problem : finding shortest paths through a weighted planar subdivision", Journal of ACM 38(11), pp.18-73, 1991.

[Pri94] Prins, C., Algorithmes de graphes, Eyrolles, 1994.

[Ros66] Rosenfeld, A., and J.L. Pfaltz, "Sequential operations in digital picture processing", Journal of ACM 13(4), pp.471-494,1966.

[Zha93] Zhan, C., S. Menon, and P. Gao, "A Directional Path Distance Model for Raster Distance Mapping”, COSIT'93, LNCS 716, Springer-Verlag, pp.434-443, 1993. 